\title{
The impact of attention load on the use of statistical information and coarticulation as speech segmentation cues
}

\author{
Tânia Fernandes \\ Universidade do Porto, Porto, Portugal \\ RÉGINE KOLINSKY \\ FNRS and Université Libre de Bruxelles, Brussels, Belgium \\ AND \\ Paulo Ventura \\ Universidade de Lisboa, Lisbon, Portugal
}

\begin{abstract}
In two artificial language learning experiments, we investigated the impact of attention load on segmenting speech through two sublexical cues: transitional probabilities (TPs) and coarticulation. In Experiment 1, we observed that coarticulation processing was resilient to high attention load, whereas TP computation was penalized in a graded manner. In Experiment 2, we showed that encouraging participants to actively search for "word" candidates enhanced overall performance but was not sufficient to preclude the impairment of statistically driven segmentation by attention load. As long as attentional resources were depleted, independently of their intention to find these "words," participants segmented only TP words with the highest TPs, not TP words with lower TPs. Attention load thus has a graded and differential impact on the relative weighting of the cues in speech segmentation, even when only sublexical cues are available in the signal.
\end{abstract}

Speech is a continuous stream, with few reliable cues about word boundaries (see, e.g., Klatt, 1980; Liberman $\&$ Studdert-Kennedy, 1978). Two major mechanisms may help solve the speech segmentation problem. First, multiple word candidates are activated by the input and compete with each other (e.g., McQueen, Norris, \& Cutler, 1994; Norris, McQueen, \& Cutler, 1995). Second, listeners exploit multiple sublexical cues probabilistically associated with word boundaries, such as subsegmental information (e.g., degree of coarticulation ${ }^{1}$ : Fernandes, Ventura, \& Kolinsky, 2007; Mattys, 2004; Mattys, White, \& Melhorn, 2005), segmental information such as transitional probabilities (TPs) between adjacent syllables, where dips in TPs are treated as likely word boundaries ${ }^{2}$ (e.g., Saffran, Aslin, \& Newport, 1996; Saffran, Newport, \& Aslin, 1996), and suprasegmental information (e.g., lexical stress; Mattys, 2004). These sublexical cues, first reported as potential sources of noise (Christiansen \& Allen, 1997), were later shown to assist language acquisition (e.g., Johnson \& Jusczyk, 2001; Thiessen \& Saffran, 2003) and to modulate lexical activation in adulthood (e.g., Davis, Marslen-Wilson, \& Gaskell, 2002; Gow \& Gordon, 1995; Salverda et al., 2007).

Mattys and colleagues (Mattys, 2004; Mattys et al., 2005) have proposed a hierarchical framework of the organization of sublexical and lexical sources of information that assist speech segmentation, in which the several types of cues are represented at three tiers. The first, top tier consists of high-level (e.g., lexical) information, which seems the most reliable information in intact listening conditions. The second, middle tier consists of segmental and subsegmental information, and the lowest tier corresponds to metrical prosody, which seems reliable in noisy listening conditions. Under this view, the involvement of any segmentation cue, either lexically driven or signal derived, is graded, since the differential weighting of the types of information available in the signal is modulated by listening conditions. This framework thus offers a much more ecological view of speech processing, because in daily communication the speech signal is often experienced under a processing load of some sort.

Recent work by Mattys, Brooks, and Cooke (2009) has shown, in addition, that the relative weighting of the available segmentation cues depends on the type of processing load, with these types being broadly categorized as perceptual load and cognitive load. Perceptual load is defined as any alteration to the signal leading to reduced acoustic integrity. Cognitive load is any load with effects arising from the recruitment of central, domain-general processing resources due to concurrent (attentional or

T. Fernandes, tfernandes@fpce.up.pt 
mnemonic) processing, rather than from a distortion of the signal (see, e.g., Cooke, Lecumberri, \& Barker, 2008). Mattys et al. (2009) proposed relating these concepts to prevalent constructs in psychophysics, specifically to the distinction between energetic masking and informational masking. Energetic masking occurs when the audibility of a target signal is reduced by a mask as a result of a blending of their acoustic signals at the periphery. Informational masking is anything that "reduces intelligibility once energetic masking has been accounted for" (Cooke et al., 2008, p. 415), and hence refers predominantly to the higher level (e.g., attentional) consequences of masking, independent of signal degradation per se. Thus, whereas energetic masking usually causes secondary informational masking (e.g., the impoverished quality of the sensory input entails a cascade of shortcomings for lexical access and sentential integration; see Mattys et al.), informational masking can occur alone.

As reviewed by Mattys et al. (2009), prior to their own work, most studies had only evaluated the impact of signal degradation through perceptual load. Only a few had addressed the impact of cognitive load on speech processing, and rather indirectly (e.g., Jacquemot, Dupoux, Decouche, \& Bachoud-Lévi, 2006; Mirman, McClelland, Holt, \& Magnuson, 2008; see also Astheimer \& Sanders, 2009). In Mattys et al.'s (2009) study, severe energetic masking decreased reliance on lexical-semantic knowledge and increased reliance on acoustic detail—namely, on acoustic cues linked to coarticulation. This result seems at odds with Fernandes et al.'s (2007) finding that background noise affected reliance on coarticulatory cues more than reliance on TP computation in artificial language learning (ALL). Yet the discrepancy between the results of Mattys et al. (2009) and those of Fernandes et al. can be explained by the higher salience of the acoustic cues used by Mattys et al. (2009). ${ }^{3}$ In particular, according to a glimpsing approach to energetic masking (cf. Cooke, 2006; see Mattys et al., 2009), an impoverished sensory input causes a cascade of shortcomings for lexical access and sentential integration. In some cases, lexical access is so disturbed by sensory degradation that listeners preferentially use whatever local salient sublexical cues can be glimpsed from the signal to segment the speech stream. This is precisely what Mattys et al. (2009) observed. In contrast, in Mattys et al.'s (2009) study, informational masking, induced by a resource-depleting competing task (either divided attention or a working memory load), resulted in the opposite pattern: It increased reliance on lexicalsemantic knowledge and was detrimental to sublexical (coarticulatory) cues.

Mattys et al.'s (2009) results thus clearly show that perceptual and cognitive load do affect differently the relative weighting of speech segmentation cues. However, they do so to the extent that lexical-semantic knowledge is involved. It remains to be investigated whether cognitive load would differentially affect the use of different sublexical cues in the absence of such higher order knowledge. The effects of perceptual versus cognitive load may depend on the type of cues at issue, as suggested by Mattys et al. (2009).
In the present study, we used an ALL setting to evaluate the impact of cognitive load on the relative weighting of two sublexical cues in the absence of lexical-semantic knowledge. ALL is adequate to examine this question, since it involves neither long-term memory representations nor access to meaning, and hence allows contrasting, in the absence of higher order knowledge, two sublexical cues as different as the statistical information afforded by (syllable-based) TPs and acoustic-phonetic coarticulatory cues.

We used exactly the same artificial language (AL) as Fernandes et al. (2007). In fact, our previous work had shown that, in ALL and with the specific coarticulatory cues we used, sensitivity to TPs survives white noise superimposition better than coarticulation does. Therefore, contrasting the effect of perceptual load with the effect of cognitive load investigated in the present study would offer a comparison similar to the one made by Mattys et al. (2009) with lexical-semantic and sublexical information.

In both Experiments 1 and 2, cognitive load was defined as the attentional cost associated with actively processing a competing source of information (here, a visual nonlinguistic task). We will thus refer to it as an attention load. Since Experiment 1 used an incidental learning condition (participants were told neither to attend to the speech stream nor that they would be subsequently tested on that material), in Experiment 2 we examined whether incidental learning could by itself penalize ALL. With this aim in mind, we compared incidental learning with an intentional learning situation in which participants were required to attend to the $\mathrm{AL}$ in order to actively search for the "word" candidates.

\section{EXPERIMENT 1}

We evaluated the impact of attention load on the relative weighting of TPs and coarticulation through three between-participants conditions differing in the number and congruence of the available cues, as in Fernandes et al. (2007). In two (between-participants) conditions of attention load, the AL was presented simultaneously with a visual stream of pictures using a 500-msec stimulus onset asynchrony (SOA) (rapid serial visual presentation, RSVP). In the low-attention-load condition, participants were passively exposed to the AL and to the RSVP, whereas in the high-attention-load condition they had to detect repetitions in the RSVP stream, a task presented as their main undertaking. According to Mattys et al. (2009), ignoring the presence of a concurrent stimulus (here, the RSVP) requires stream segregation and selective attention and hence induces some informational masking in both attentional conditions, but higher cognitive load would be involved only when participants have to process this concurrent stimulus (here, in the high-attention-load condition), because this requires sharing limited processing resources.

The adoption of a concurrent visual as opposed to an auditory task offers two main advantages. First, it guarantees that the pattern of ALL cannot be due to energetic masking of the signal. Second, using the same RSVP task 
in an ALL setting, Toro, Sinnett, and Soto-Faraco (2005) found a dramatic impairment in TP extraction, which did not occur with a longer (750-msec) SOA. This shows that statistical speech segmentation is affected by a severe depletion of attentional resources.

We also examined whether attention load differentially modulates the relative weighting of TPs and coarticulatory cues. If the impact of such a cognitive load on the weighting of these cues resembled that of perceptual load, the overall pattern of results would echo the one reported by Fernandes et al. (2007) - namely, resilience of statistical information coupled with reduction of coarticulation reliability in high attention load. However, according to Mattys et al. (2009; Mattys et al., 2005), lower weighted cues are more demanding with regard to central resources. Although TPs and coarticulatory cues are represented at the same (middle) tier in the hierarchical framework proposed by Mattys (2004; Mattys et al., 2005), Fernandes et al. demonstrated that as long as the quality of the signal is intact, coarticulatory cues outweigh TPs (see also infant data in Johnson \& Jusczyk, 2001), which suggests that the latter tax central resources more than the former. If this were true, statistically driven speech segmentationwhich is very resilient to energetic masking - would on the contrary be more affected by attention load than by coarticulation.

As with any other form of impoverishment, attention load could also have a graded rather than an all-or-none impact on the weighting of speech segmentation cues: The available cues would not be simply "switched off" in impoverished conditions; instead, they would gradually lose power while other cues remain resilient to masking. This idea was evaluated by using AL words with different levels of TPs: Half were high-TP words (TP level ranging from .75 to 1) and the others were low-TP words (TP level ranging from .50 to .58$)$. If attention load had a graded impact on TP computation, it would affect mainly or perhaps only the extraction of low-TP words, not (or less so) the extraction of high-TP words. In this case, a TP gradient would be found only in high attention load, with better performance for high- than for low-TP words.

\section{Method}

\section{Participants}

Eighty-six undergraduate psychology students at the Universities of Lisbon and Evora, who were monolingual European Portuguese speakers, participated in the experiment for course credit. Forty-one were randomly assigned to the low-attention-load condition (13 in the TPs-only condition, 14 in the congruent cues condition, and 14 in the incongruent cues condition), and 45 to the high-attention-load condition (15 in each one of the three cue type conditions).

\section{Material}

AL material. To achieve realistic coarticulation, we used naturally produced utterances rather than synthesized speech, and created concatenated and coarticulated versions of the AL stimuli (for the detailed method, see Fernandes et al., 2007). On the basis of Fernandes et al.'s pretests, we already know that the use of naturally produced rather than synthesized utterances does have an impact on ALL performance.

All the natural speech stimuli were recorded in a soundproof room (using WaveLab Lite Program, version 2.52) by a European Portuguese female native speaker using a Shure M58 microphone and sampled at a rate of $22.05 \mathrm{kHz}$ with 16-bit conversion. Adobe Audition 1.5 and Praat 4.2.24 (available at http://fon.hum.uva.nl/ praat/) were used for editing of the digitized versions of the stimuli (i.e., syllables, words, and part-words). These stimuli were closely matched to a synthesized stream in all relevant acoustic parameters, such as speaking rate (approximately 270 syllables per minute), AL stimuli duration, lack of lexical stress cues to word boundaries, and pitch contour (through flattening to monotone $220 \mathrm{~Hz}$ ).

The AL used by Fernandes et al. (2007) was adopted here. It was constituted by six trisyllabic AL words (i.e., /bebuku/, /bukele/, /lufebe/, /kefubi/, /fufibu/, /kilebu/). TPs between adjacent syllables were always higher within than between $\mathrm{AL}$ words. Half of the AL words had higher TPs (high-TP words, ranging from .75 to 1) than the other half (low-TP words, ranging from .50 to .58), but all occurred with the same frequency in the AL stream.

For the familiarization phase, three versions of the AL were created. Each version included the same sequence of syllables, divided into three listening blocks of approximately $7 \mathrm{~min}$ each (i.e., $21 \mathrm{~min}$ total exposition). Each block was created by concatenating 105 tokens of each of the six AL words (1,890 syllables, 630 tokens of words), with the criterion that two tokens of the same word never occurred adjacent to each other in the stream.

As illustrated in Table 1, the only difference between the three versions of the AL was the type of information available in the speech stream. In the TPS-only condition, only the concatenated version of the AL was used: Each CV syllable that constituted the AL inventory was recorded individually (thereby avoiding coarticulation between syllables) and then concatenated to form the AL stream; hence, the only available segmentation cues were the TPs between syllables. For the other two versions, besides concatenated portions, there was also coarticulated AL material. The same number of coarticulated exemplars occurred in the congruent cues and the incongruent cues conditions, the only difference being the stimuli that appear in their coarticulated form: the AL words in the congruent cues condition, and the part-words in the incongruent cues condition. These coarticulated exemplars were located as closely as possible in the speech stream in these two versions of the AL.

The forced choice test phase included the six AL words and the six part-words in their concatenated version. Part-words consisted of syllables of two different AL words that had appeared adjacently in the stream, and that hence have a lower TP level than TP words do. Three part-words were constituted by the last syllable of one AL word and the first two syllables of the next AL word (see Part-Words $1 \# 2$, in Table 1). The other three part-words were constituted by the last two syllables of an AL word and the first syllable of the next AL word (see Part-Words 2\#1, in Table 1). The same test stimuli and forced choice test were presented to all participants.

Table 1

Orthographic Translation of an Artificial Language Stream Sample

\begin{tabular}{llcc}
\hline \multicolumn{1}{c}{ Cue Type } & \multicolumn{1}{c}{ Speech Stream } & Part-Words 1\#2 & Part-Words 2\#1 \\
\hline TPs only & lu-fa-ba-\#ki-la-bu-\#ka-fu-bi-\#ba-bu-ku-\#bu-ka-la-\#fu-fi-bu ... & ba-\#ki-la & ka-la-\#fu \\
Congruent cues (Coarticulated TP words) & LUFABA-\#ki-la-bu-\#ka-fu-bi-\#ba-bu-ku-\#bu-ka-la-\#FUFIBU $\ldots$ & BA-\#ki-la & ka-la\#\#U \\
Incongruent cues (Coarticulated part-words) & lu-fa-BA\#KILA-bu-\#ka-fu-bi-\#ba-bu-ku-\#bu-KALA\#FU-fi-bu ... & $\underline{\text { BA\#KILA }}$ & $\underline{\text { KALA\#FU }}$ \\
\hline
\end{tabular}

Note-The "\#” defines word boundaries according to transitional probabilities; the "-" represents concatenation; coarticulated syllables are presented in capitalized letters and underlined. 
RSVP material. For the RSVP, we selected 12 pictures of three semantic categories (4 pictures per category) from Snodgrass and Vanderwart (1980). We converted the pictures into .bmp files and created rotated exemplars $\left(30^{\circ}\right.$ to the right or to the left) with Irfanview (available at www.irfanview.com). The RSVP occurred simultaneously with the AL presentation during the familiarization phase. We created three RSVP blocks (with stimuli blocked according to semantic category) of 832 trials each so they would have a similar duration as the three AL blocks. Within each block, the 4 pictures appeared in both rotations and with the same frequency of occurrence. Repetitions of the same picture (independently of the type of rotation) occurred in approximately $25 \%$ of the trials. Visual stimuli were presented centrally at the same spatial location for $250 \mathrm{msec}$ each, with an ISI of $250 \mathrm{msec}$, in a sequence order (SOA of $500 \mathrm{msec}$ ). The RSVP was displayed on an HP Compaq 7500 computer monitor with a 17 -in. screen. The order of the three visual blocks was randomized for each participant.

\section{Procedure}

Participants were tested individually or in groups of two in a sound-attenuated room. They were seated approximately $50 \mathrm{~cm}$ away from the computer screen. In the familiarization phase, auditory stimuli were presented using Windows Media Player. All the auditory stimuli were presented at a comfortable volume level through Sennheiser HD 280 Professional Silver headphones. For the RSVP and for the forced choice test phase, presentation, timing, and data collection were controlled by E-Prime 1.1 (Schneider, Eschman, \& Zuccolotto, 2002a, 2002b).

All participants were informed that the experiment had two phases. In the first phase they were presented with an incidental ALL condition: They were told that they would hear an AL (no information about the AL was given) and would simultaneously see rotated pictures, presented one at a time on the computer screen. Nothing was said about the second (forced choice test) phase of the experiment.

In the low-attention-load condition, participants were asked to passively listen to and watch the stimuli that would be presented. They were told that the experiment was aimed at evaluating any possible effect of passive exposition to neutral auditory and visual stimuli on reading comprehension of a subsequent text. They were informed that the text they would read later on had no direct relation to the auditory and visual stimuli presented in the first phase. These instructions aimed at forcing participants to allocate their attention to both the visual and auditory streams, in a nonetheless incidental passive learning situation (see Turk-Browne, Jungé, \& Scholl, 2005).

In the high-attention-load condition, participants' attention was diverted to the visual stream, and the AL was presented as an irrelevant distractor. They were asked to press the "1" key on the keyboard with their dominant hand as soon as they detected a repetition between two consecutive visual stimuli, independently of their orientation. Correctness on visual detection was emphasized.

In the second phase, all participants performed the same forced choice test. They were told that, although they were not asked to attend to the AL presented in the first phase, this new language was constituted of words and that their potential knowledge about these $\mathrm{AL}$ words was going to be evaluated. They were asked, on each trial, to indicate which of the two strings presented (always an AL word and a part-word) sounded more like a word from the new language. They indicated their responses by pressing the " 1 " or " 2 " key on the keyboard, depending on whether they chose the first or the second string, respectively. Each trial started with a warning tone, followed by two trisyllabic strings separated by $500 \mathrm{msec}$ of silence, presented through headphones. Each AL word was paired exhaustively with each part-word in a total of 36 trials. Immediately after a participant gave his or her answer, or if no answer was registered after a maximum of $10 \mathrm{sec}$, another trial began. Participants were asked to answer on all the trials, even if they were not totally sure about their decision, but accuracy of response was also emphasized. Order of presentation of trials was randomized for each participant, and order of presentation of the AL stimuli within trials was counterbalanced within group.

Four practice trials were given prior to the test, in order to enable practice with keypresses. On each practice trial, two sounds (animal and environmental) were presented; participants were asked to decide which one was the animal sound. Feedback on correctness of response was provided only for these practice trials.

After the forced choice test, participants in the high-attention-load condition were dismissed, and participants in the low-attention-load condition silently read a text and answered four questions about it.

\section{Results and Discussion}

\section{Detection of Repetition in RSVP}

For the three high-attention-load groups, which all had to detect repetitions in the RSVP, false alarms (FAs) and hits were combined to calculate $d^{\prime}$ scores, separately for each participant, cue type, and block (see Table 2). The ANOVA performed on $d^{\prime}$ scores with cue type and block as between-participants factors revealed no significant effect or interaction (all $F \mathrm{~s} \leq 1$ ). In addition, for each cue type the average $d^{\prime}$ score was significantly greater than zero [all $t \mathrm{~s}(14)>12.2, p \mathrm{~s}<.0001]$. Participants were thus able to discriminate the situation in which a repetition occurred from that in which it did not, and they did so at the same success level in the three cue type conditions. Thus, any effect of cue type that may be observed in the ALL pattern cannot be due to attention load differences between the high-attention-load groups.

\section{ALL Performance}

A mixed ANOVA with attention load (low vs. high) and cue type (TPs only, congruent cues, incongruent cues) as between-participants factors, and TP level (low- vs. highTP words) as the within-participants factor was run over

Table 2

Experiment 1: Hits and False Alarms (FAs) for the Three Cue Type Conditions in the RSVP Task (in High Attention Load), According to Visual Block and $d^{\prime}$ Scores

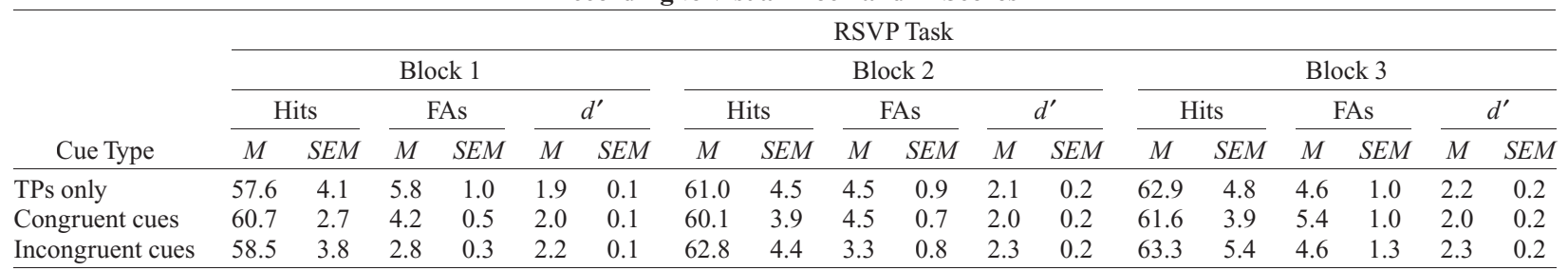


the raw number of TP-word choices, the average values of which are reported in Table 3.

The main effect of cue type was significant $[F(2,80)=$ $\left.46.31, M S_{\mathrm{e}}=6.93, p<.0001\right]$. Participants in the congruent cues condition had the best ALL performance in comparison with the TPs-only and the incongruent cues conditions $[F(1,80)=9.18$ and 89.05 , respectively, both $p s<.005]$. Only participants exposed to either TPs only or congruent cues presented above-chance performances showing that they were able to extract the AL words from the stream (see Table 3). In contrast, with incongruent cues, participants more often chose the coarticulated partwords over TP words as the units of the new language, which led to a below-chance performance that differed significantly from that of listeners in the TPs-only condition $[F(1,80)=39.96, p<.0001]$.

No significant main effect of attention load $[F(1,80)=$ $\left.2.17, M S_{\mathrm{e}}=6.93, p=.14\right]$ or of TP level $(F<1)$ was found, but the interaction between attention load and TP level was significant $\left[F(1,80)=4.00, M S_{\mathrm{e}}=6.65, p<\right.$ .05]. Indeed, performance for high-TP words was unaffected by attention load $(F<1)$, but attention load did penalize low-TP-words parsing: For low-TP words, ALL performance was worse in high as opposed to low attention load $\left[F(1,80)=6.7, M S_{\mathrm{e}}=7.51, p=.01\right]$. The interaction between attention load and TP level did not vary significantly as a function of cue type $(F<1.5)$.

With regard to TP computation, this pattern of results is thus opposite to the one found under perceptual load (Fernandes et al., 2007): Attention load affects statistically driven speech segmentation in a graded manner. Indeed, in high attention load, whereas salient statistical units were still extracted from the stream, the extraction of less salient ones was penalized.

Before drawing a definitive conclusion, however, one should check whether these results are not (at least partly) due to the specific incidental learning condition used. Indeed, participants were told neither to attend to the speech stream nor that they would be tested subsequently on this material. Thus, this was not a genuine dual-task (or divided-attention) situation, and one may hence wonder whether incidental learning by itself, or in combination with attention load, could have penalized the extraction of TPs. In Experiment 2, we thus examined whether the pattern of results would be affected by the fact that partici- pants were required to attend to the AL (in an intentional learning condition) or not (in incidental learning).

\section{EXPERIMENT 2}

The observation that speech segmentation did succeed in an incidental learning situation such as the one used in Experiment 1 is by itself interesting. It is in line with previous results showing that statistically driven segmentation can occur even in conditions in which attention is not fully allocated to the AL stream (e.g., Toro et al., 2005; see also Saffran, Newport, Aslin, Tunick, \& Barrueco, 1997, who observed ALL with a concurrent drawing task), and with listeners who cannot be formally instructed to attend to the speech stream (human infants - see, e.g., Saffran, Aslin, \& Newport, 1996-or nonhuman primates-see, e.g., Hauser, Newport, \& Aslin, 2001). More generally, these results converge with evidence showing that the speech signal (or other dynamic and complex auditory stimuli; see, e.g., Jones, Macken, \& Murray, 1993) is processed even when participants are asked to ignore it, as demonstrated, for example, by the irrelevant sound effect (or irrelevant speech effect, cf. Colle \& Welsh, 1976; for a review, see, e.g., Jones, 1999) observed in short-term memory tasks and more recently in visual statistical learning (Neath, Guérard, Jalbert, Bireta, \& Surprenant, 2009).

The resilience to high attention load of coarticulationdriven segmentation in the incidental learning situation used in Experiment 1 suggests that this segmentation procedure is less attention demanding than the computation of statistical information. However, informational masking (or cognitive load) has multiple facets (Cooke et al., 2008). For example, Pacton and Perruchet (2008) recently showed that learning depends on attentional involvement even in incidental learning: Although participants were neither required to search for the statistical regularities of the sequences nor to explicitly memorize them, when they were faced with materials embedding relations between both adjacent and nonadjacent elements, they did not learn adjacent dependencies when the instructions drew their attention toward nonadjacent dependencies, and vice versa. Thus, attentional allocation must be distinguished from intention to learn (or "implicitness," according to Pacton \& Perruchet, 2008).

Table 3

Average Proportions of Artificial Language (AL) Word Responses (in Percentages), Separately for Each Cue Type and Attention Load Condition, Considering Transitional Probability (TP) Level of AL Words (High- vs. Low-TP Words), and Local One-Way $t$ Test (in Comparison With Chance Level, 50\%)

\begin{tabular}{|c|c|c|c|c|c|c|c|c|c|}
\hline \multirow[b]{4}{*}{ Cue Type } & \multicolumn{9}{|c|}{ Attention Load } \\
\hline & \multicolumn{4}{|c|}{ Low } & \multicolumn{4}{|c|}{ High } & \multirow[b]{3}{*}{ Average } \\
\hline & \multicolumn{2}{|c|}{ High-TP Words } & \multicolumn{2}{|c|}{ Low-TP Words } & \multicolumn{2}{|c|}{ High-TP Words } & \multicolumn{2}{|c|}{ Low-TP Words } & \\
\hline & $M$ & $S E M$ & $M$ & $S E M$ & $M$ & $S E M$ & $M$ & $S E M$ & \\
\hline TPs only & 57.2 & $3.6^{*}$ & 62.8 & $3.2^{* *}$ & 65.5 & $3.3^{* *}$ & 52.2 & 3.8 & $59.4^{*}$ \\
\hline Congruent cues & 65.8 & $3.1^{* *}$ & 73.4 & $3.7^{* *}$ & 63.7 & $3.5^{* *}$ & 68.1 & $5.6^{* *}$ & $67.7^{* *}$ \\
\hline Incongruent cues & $\underline{44.0}$ & $\underline{3.8}^{(*)}$ & 45.2 & $\underline{3.6}^{(*)}$ & $\underline{41.1}$ & $\underline{3.9}^{*}$ & $\underline{38.1}$ & $\underline{3.8}^{* *}$ & $\underline{42.1}^{*}$ \\
\hline
\end{tabular}

Note-Underlined scores refer to below-chance level and hence reflect preference for coarticulated part-words over TP words. $\left.\quad{ }^{*}\right) p \leq .10 . \quad{ }^{*} p<.05 . \quad{ }^{* *}$ at least $p<.01$. 
Likewise, attention load and intention to learn may have different and perhaps additional effects on ALL. Their joint action may, for example, explain why in Saffran and colleagues' studies, ALL performance was much lower (only $59 \%$ ) when participants performed a resource-consuming task in incidental learning (Saffran et al., 1997) than when there was no diverting task and learning was intentional (in which case performance reached 76\%; Saffran, Newport, \& Aslin, 1996).

It is thus possible that high attention load affects statistically based speech segmentation only in incidental learning, not in intentional learning. Here, to evaluate this possibility, we directly compared these two learning conditions. Intentional learning actually corresponded to a dual-task condition, which may be similar to everyday situations in which speech is occasionally processed at the same time as a nonlinguistic nonauditory distractor, such as driving a car while talking to someone, listening to the phone while working on one's computer, or preparing dinner while listening to the news on the radio. The comparison between the two learning conditions was, however, restricted to TP computation, since no detrimental effect of attention load on the use of coarticulatory cues was observed in Experiment 1. We predicted, in particular, that if intentional learning enabled listeners to use statistical information efficiently, they would segment low-TP words as well as high-TP words, even in high attention load.

\section{Method}

\section{Participants}

Forty-eight new undergraduate psychology students at the University of Lisbon participated in the experiment for course credit; 22 were randomly assigned to the low-attention-load condition (13 in incidental learning, 9 in intentional learning) and 26 to the highattention-load condition (13 in each learning condition).

\section{Material}

The same AL in the TPs-only condition of Experiment 1 was adopted here, but using synthesized speech (text-to-speech MBROLA software-Dutoit, Pagel, Pierret, Bataille, \& van der Vrecken, 1996with a European Portuguese female diphone database: http://tcts fpms.ac.be/synthesis/mbrola.html). The three 7-min visual blocks of Experiment 1 were also used. The 36 trials of the forced choice task were prepared as in Experiment 1 but using synthesized speech.

\section{Procedure}

All participants were exposed to the three AL and visual blocks (lasting a total of $21 \mathrm{~min}$ ). The same procedure as in Experiment 1 was used in the incidental learning condition (both for low- and high-attention-load groups); only the instructions given to the two intentional learning groups differed. The latter groups were informed that the AL comprised a limited number of "words" and that their knowledge of those "words" was going to be evaluated in a subsequent phase. They were thus presented with a dual-task situation: They were asked to simultaneously attend to the $\mathrm{AL}$ and to the visual stream. Instructions about the AL and the forced choice test were, however, quite vague; that is, no information on any characteristic of the $\mathrm{AL}$ was given.

The low-attention-load intentional learning group was simply asked to passively attend to both the visual and the auditory streams, since they would have to perform two subsequent tasks on those materials: one about their knowledge of the AL words and the other about their rotational preference for the visual material.
The high-attention-load intentional learning group was asked to attend to the AL while performing the visual repetition detection task. Both correctness in the visual detection task and importance of attending to the AL were emphasized, so that participants would correctly perform the second-phase task. To provide incentive so that participants would, in fact, be in a condition of high attention load, participants with the best performances in the visual task were given a voucher for a local bookstore.

After the first phase, all participants performed the AL twoalternative forced choice test.

\section{Results and Discussion \\ Detection of Repetition in RSVP}

For the two high-attention-load groups, FAs and hits of the repetition detection task were combined for calculating $d^{\prime}$ scores (as in Experiment 1) separately for each participant in each learning condition. The average $d^{\prime}$ scores of the two groups were equivalent $[t(24)=1.15$, $p>.10]$ and significantly greater than zero, with scores of $1.95(S E=0.17)$ for incidental learning $[t(12)=11.30$, $p<.0001]$ and $1.70(S E=0.13)$ for intentional learning $[t(12)=12.69, p<.0001]$. This pattern of results ensures that any difference to be found in ALL patterns between these two groups cannot be attributed to differences in the depletion of attentional resources.

\section{ALL Performance}

In order to evaluate the impact of both attention load (low vs. high) and learning condition (incidental vs. intentional) on statistically driven segmentation, we ran a mixed ANOVA with these two between-participants factors and TP level (high- vs. low-TP words) as a within-participants factor over the raw number of AL word choices.

Only the main effect of learning condition was significant $\left[F(1,44)=4.6, M S_{\mathrm{e}}=4.48, p<.05\right.$; attention load and TP level: both $F_{\mathrm{s}}(1,44)<1.5$ ], with better ALL in intentional than in incidental learning (on average, $65.1 \%$ vs. $59.7 \%$, respectively). ALL was thus facilitated when participants' attention was directed to the speech stream.

However, this facilitation did not compensate for the cost induced by the depletion of attentional resources. Indeed, as illustrated in Figure 1, there was a significant interaction between attention load and TP level $[F(1,44)=$ $\left.8.7, M S_{\mathrm{e}}=6.77, p=.005\right]$, which was not modulated by learning condition $(F<1)$. As was the case in Experiment 1 , only the extraction of low-TP words was severely affected by attention load $[F(1,44)=9.7, p<.005]$, not that of high-TP words $[F(1,44)=2.0, p=.17]$. Indeed, whereas in low attention load participants were able to extract both low- and high-TP words above chance [on average, $66.6 \%$ and $61.3 \%$, respectively; $t(21)=6.3$ and 3.7 , respectively; both $p \mathrm{~s}<.005$ ]; in high attention load they were only able to extract the high-TP words above chance [on average, $67.1 \%, S E=3.6, t(25)=6.3, p<$ .001 ], not the low-TP words [on average, 54.7\%, $S E=$ $3.9, t(25)=1.7, p=.11]$. There was thus a significant TP gradient only in high attention load $[F(1,44)=9.5, p<$ $.005]$, not in low attention load $(F=1.4)$.

In sum, ALL was penalized in a graded way by a depletion of attention resources, independently of the fact that 


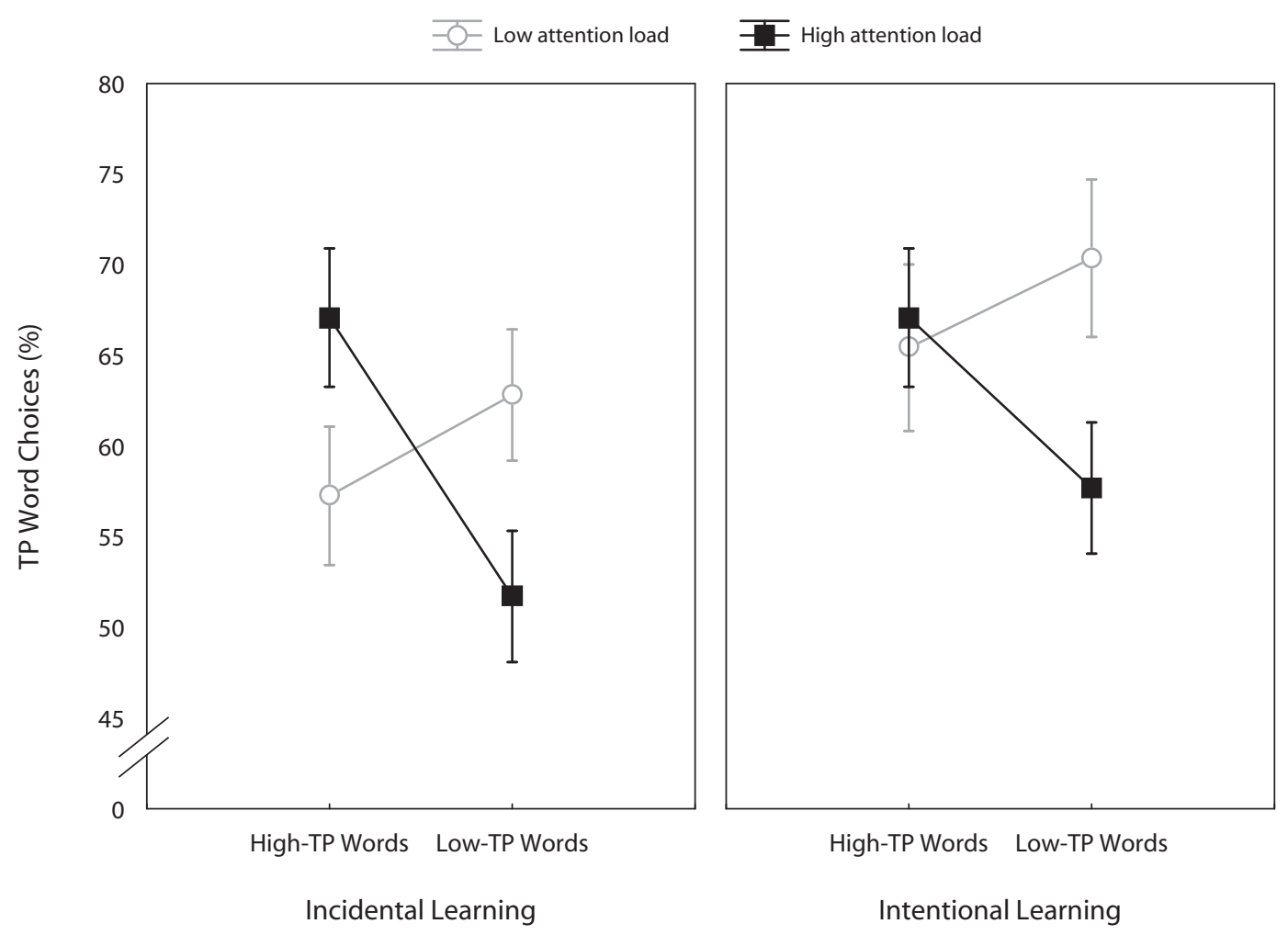

Figure 1. Experiment 2: Proportion of transitional probability (TP) word responses broken down by TP level of the TP words, in each learning (incidental vs. intentional) condition and attention load (low vs. high). Vertical bars denote standard errors of the means in each condition (chance level, $50 \%$ ).

participants were engaged (or not engaged) in the active search of the AL "words." Indeed, although such an active search did favor learning overall, the impact of attention load on statistically driven speech segmentation found in intentional learning was not different from the one found in incidental learning.

\section{GENERAL DISCUSSION}

According to a recent model of speech segmentation (Mattys, 2004; Mattys et al., 2005), the involvement of any segmentation cue, either lexically driven or signal derived, is graded: The types of information available in the signal present differential weightings that are modulated by the listening conditions. Recent work by Mattys et al. (2009; Mattys et al., 2005) showed in addition that listening conditions do not refer only to the signal quality or "perceptual load," but also to cognitive load due to concurrent attentional or mnemonic processing, leading to what these authors call informational masking.

The impact of perceptual load on the relative weighting of various speech segmentation cues had already been demonstrated (Fernandes et al., 2007; Mattys, 2004; Mattys et al., 2005). Mattys et al. (2009) showed that when the physical signal is intact, informational masking linked to cognitive load also affects the cues predominantly used in speech segmentation, but in a different way than perceptual load does. In noisy environments, segmentation cues that are perceptually more salient have a higher probabil- ity of being glimpsed (cf. Cooke, 2006), and hence exhibit greater segmentation power than less salient cues. In contrast, increased reliance on higher order knowledge would be a generalized response to cognitive load, which would be particularly detrimental to segmentation cues that have a secondary role in speech segmentation. In other words, besides being weighted lower in speech segmentation, sublexical cues would also be more demanding on central resources.

Nevertheless, it was hitherto unclear whether cognitive load would also have a differential impact on the relative weighting of different sublexical segmentation cues when no higher level information may assist speech segmentation.

In Experiment 1, using an incidental ALL setting, we investigated the impact of attention load on the weighting of two sublexical segmentation cues: TPs, a statistical segmental information (here related to syllables), and coarticulation, a local subsegmental information.

The relative weighting of TPs and coarticulation varies as a function of physical quality of the signal (Fernandes et al., 2007). With phonetically intact speech, coarticulation is a powerful segmentation cue, prevailing over other available sublexical cues (TPs_-Fernandes et al., 2007; lexical stress-Mattys, 2004; Mattys et al., 2005). On the other hand, at least with the very same material as is used in the present study, coarticulation is much more affected by white noise superimposition than are TPs, which are highly resilient to it (Fernandes et al., 2007). Therefore, 
contrasting the effect of attention load (investigated in the present study) with the effect of perceptual load previously reported by Fernandes et al. offered a comparison similar to that made by Mattys et al. (2009) with lexicalsemantic and sublexical information.

The results of Experiment 1 showed that the impact of attention load on the relative weighting of TPs and coarticulation was qualitatively different from that of perceptual load - that is, of white noise superimposition (Fernandes et al., 2007). Contrary to what was found with perceptual load, in the high-attention-load condition coarticulation was able to drive the segmentation process when statistical information suggested different, incompatible segmentation hypotheses. In contrast, the ability to adopt a statistically based speech segmentation procedure was severely affected by cognitive load. The higher resilience to cognitive load of coarticulation-driven segmentation in comparison with TP computation suggests that the former procedure is less attention demanding than the latter. This is coherent with Mattys et al.'s (2009) proposal that cues that are weighted lower in optimal listening conditions (i.e., with no perceptual or cognitive load) are more demanding on central resources.

However, the negative impact of attention load on the statistical segmentation procedure could merely stem from the fact that in the high-attention-load condition of Experiment 1 the AL was not the central focus of the participants' attention. It could thus be the case that TP computation would operate efficiently (with no impact of a severe depletion of attention resources) if attention were directed to the speech stream, as would be the case in an intentional learning setting. The pattern of results of Experiment 2, which used both an intentional and an incidental learning condition, demonstrates that directing the focus of attention to the AL by pushing participants to actively search for AL "words" candidates did modulate overall performance (with better performance in intentional than in incidental learning), but was not sufficient to preclude the impairment of statistically driven segmentation by attention load. Indeed, this impairment was similar whether participants were attending to the AL (in intentional learning) or their attention was diverted from the AL (in incidental learning).

In addition, as previously found for perceptual load (Fernandes et al., 2007; Mattys et al., 2005), in both experiments of the present study attention load had a graded rather than an all-or-none impact on speech segmentation. In the high-attention-load TPs-only condition, only highTP words, but not low-TP words, were correctly extracted from the stream, and they were extracted at the same level as in low attention load. Thus, as proposed by Mattys et al. (2009; Mattys et al., 2005), the cues more susceptible to a specific load (e.g., TPs to attention load) are not simply "switched off."

Another aspect deserves further comment. On the basis of their own results, Mattys et al. (2009) argued against a simple, compensatory account of the differential weighting of speech segmentation cues under perceptual or cognitive load. According to this account, which they called the resource trade-off hypothesis, the less one type of cue is used, the more other available cues gain importance. The pattern of results found in the present study regarding cognitive load and the data reported by Fernandes et al. (2007) do not seem to cohere with the resource trade-off hypothesis, either. In Fernandes et al., speech segmentation driven by coarticulation was strongly penalized by energetic masking, but TPs alone did not gain power with the increase of perceptual load. Indeed, for participants exposed only to statistical information, there was no gain in performance with the increase of energetic masking; and for those exposed to congruent cues, performance dropped as a function of signal quality. Thus, TP computation seems merely to resist energetic masking, maintaining similar power in driving speech segmentation under intact and degraded conditions, but is unable to compensate for the unreliability of coarticulation due to energetic masking. The resource trade-off hypothesis is less easy to evaluate in the present study, since no condition included only coarticulatory cues. Still, in Experiment 1 , the impairment of TP computation by attention load did not seem to be compensated by a stronger undertaking of coarticulatory cues. Had this been the case, we would have observed a significant interaction between attention load, TP level, and cue type, and in particular a huge increment in using coarticulation for low-TP words extraction in the incongruent cues condition. In sum, the relative weighting of speech segmentation cues under load does vary, but not according to a simple trade-off by which when one cue loses weight, the others gain importance. What seems to matter is whether one particular cue is more resistant to the current adverse condition, and hence maintains its power at different levels of degradation, whereas other cues are strongly affected by that degradation. This seems to hold true with any type of load, either cognitive or perceptual, at least for the sublexical cues studied here.

More generally, the observation of successful statistical learning in high attention load for high-TP words may seem at odds with the fact that Toro et al. (2005) reported no TP extraction at all in an ALL setting using similar high-attention-load conditions. Since we observed highTP words segmentation in high attention load in both intentional and incidental learning, and hence independently of the AL stream being the focus of the participants' attention (or not), intention to learn cannot be the explanatory factor of this discrepancy. It cannot be attributed to cross-study differences in the attention load induced by the RSVP task either, since in the present study all the groups in the high-attention-load condition displayed an RSVP performance level very similar to that reported by Toro et al. Rather, this discrepancy may be attributed to the much longer familiarization phase we used, actually three times longer $(21 \mathrm{~min})$ than that in Toro et al. $(7 \mathrm{~min})$. As acknowledged by these authors, divided attention may not completely prevent, but only slow down, statistical learning. When taken together with Toro et al.'s data, the present results suggest that this is indeed the case, at least for high-TP words. Further work should aim at evaluating more systematically the impact of amount of familiarization on TP-driven speech segmentation. 
In any case, the graded impact of attention load on TP computation is probably not unique, because it may hold true for other sequence learning effects studied within the framework of implicit learning, with which TP-based ALL actually shares many features (for a discussion, see, e.g., Perruchet \& Pacton, 2006). Sequence learning is also an attention-dependent mechanism (Pacton \& Perruchet, 2008; Rowland \& Shanks, 2006; Shanks, Rowland, \& Ranger, 2005). Sequences with unique associations in which one event is positioned to always be followed by another event (which hence have TPs of 1) tend to be learned even under dual-task conditions, whereas sequences with lower TPs (e.g., .5) are learned poorly (Cohen, Ivry, \& Keele, 1990). Our results suggest that the same holds true in ALL. Future work should study more closely the resemblance between ALL and other sequence learning effects.

Future studies should also aim at examining what exactly are the factors underlying the differential impact of cognitive (the present study) and perceptual (Fernandes et al., 2007) load on the relative weighting of coarticulation and TPs. In addition to their intrinsic weight, the reliability of these two cues could also depend on their domain specificity. In particular, the resilience of coarticulatory cues (in comparison with TPs) to attention load could reflect the mandatory operation of an encapsulated system (cf. Fodor, 1983, 2000). Indeed, both segmentation cues are available throughout linguistic development (i.e., since an early age- - e.g., Johnson \& Jusczyk, 2001; Kirkham, Slemmer \& Johnson, 2002; Saffran, Aslin, \& Newport, 1996 - and in adulthood-e.g., Mattys, 2004; Saffran, Newport, \& Aslin, 1996), but only coarticulation is speech-specific, and hence may be processed by an encapsulated system that may operate quite independently of attention resources. On the contrary, TP computation relies on a domain-general learning mechanism (e.g., Fiser \& Aslin, 2001; Perruchet \& Pacton, 2006; Turk-Browne et al., 2005) that may be more sensitive to attention resources and hence to cognitive load. A recent study by Astheimer and Sanders (2009) also suggests that domain specificity could play a role in the allocation of selective attention to word onsets. In their study, whereas linguistic, and hence speech-specific, probes (e.g., the syllable "ba") were able to modulate listeners' selective attention to word onsets in a continuous speech flow, no impact of general auditory, nonlinguistic probes (i.e., pure tones) was found. Although the notion that domain specificity could mediate the relative weighting of these cues remains for the moment highly speculative, it could be supported if other speech-specific cues (e.g., phonotactics) were put against TPs under cognitive load and if the same relative weighting as that observed in Experiment 1 were to be found.

Another future challenge will be to specify the exact conditions under which the effects of perceptual and cognitive noise diverge. As a matter of fact, in Experiment 1, whereas in high attention load coarticulation was more reliable than TPs, in low attention load coarticulation was not powerful enough to override the incongruent statistical information. This is an odd result since coarticulation is more preponderant than TPs in speech segmentation with intact signal (Fernandes et al., 2007), and hence the same preponderance was expected in low attention load. Note, however, that data on other segmentation cues are in line with this observation. With moderated energetic masking, no preponderance of lexical-semantic information, coarticulation, or metrical prosody was found (Mattys et al., 2005, Experiment 6B). Also, Mattys et al. (2009) observed greater reliance on coarticulation over lexical information only in strong energetic masking conditions, whereas mild energetic masking equally reduced reliance on both cues. Thus, whatever the type of load or masking (either energetic or informational), segmentation does not seem to be always consistently driven by one particular cue when other conflicting cues are still sufficiently available to compete with it. This may be due to the fact that the incongruent cues condition is by itself attentionally demanding: Although incongruent cues are useful to isolate the specific contributions of each cue type and its reliability in various listening conditions, they rarely occur in natural situations.

Related to this issue, it would also be important to evaluate the role of process sharing as opposed to that of content sharing between the speech signal and a concurrent (irrelevant) distractor in the study of speech segmentation processing under cognitive load. It could be the case that the interference-by-process hypothesis of Dylan Jones and colleagues (e.g., Jones, 1999; Jones et al., 1993; Marsh, Hughes, \& Jones, 2009) would extend its application beyond working memory to the speech domain.

With regard to the impact of process sharing between critical and irrelevant materials, Neath et al. (2009) had already shown that visual statistical learning can be penalized by the simultaneous presentation of irrelevant speech. Indeed, in their study, visual TP computation was penalized only when the irrelevant speech stimuli corresponded to a changing auditory stream constituted by different letters, not when they remained unchangedthat is, when only one letter was continuously played. Our statistical learning results thus provide a mirror image of Neath et al.'s results. Whereas in that study, visual TP computation was penalized by the concurrent presentation of changing speech material, in the present study speech segmentation afforded by TP computation was penalized by the concurrent presentation of a visual stream (RVSP) of pictures. These results could be in line with the interference-by-process hypothesis (e.g., Jones, 1999; Jones et al., 1993; Marsh et al., 2009), in that both visual sequence and speech material would require seriation, which resulted in interference in processing of the critical material (e.g., the visual sequence in Neath et al., 2009; the speech material in the present study). Accordingly, the key determinant of disruption in spoken word processing could be the extent to which both the irrelevant, distractor information and spoken word processing share similar processes. Further work should test this proposal.

Finally, it should be noted that since no lexical or semantic information was ever available in the material used, the present results only demonstrate that cognitive load has a differential impact on the relative weighting of sublexical cues in the absence of such higher level cues. Hence, they cannot directly argue in favor of Mattys et al.'s (2009) pro- 
posal that cognitive load, of whatever nature, offsets the weights of sublexical segmentation cues in favor of higher level, lexically driven strategies. Nevertheless, both Mattys et al.'s (2009) work and our own studies on perceptual (Fernandes et al., 2007) and cognitive (the present study) load illustrate that if cognitive psycholinguistics' goal is to achieve the comprehension of speech segmentation processing in natural settings, the study of intact speech conditions is unsatisfactory, and that the study of "noisy" conditions should no longer be restricted to energetic masking. Indeed, both sets of studies show that cognitive and perceptual load have a differential impact on the relative weighting of the available segmentation cues.

In future work, this new approach will allow linking results obtained with laboratory-degraded speech (either from energetic or informational masking) to data observed with naturally occurring degraded speech, including with regard to the informational consequences of energetic masking. For example, studies on the cognitive impact of hearing loss in elders (e.g., McCoy et al., 2005; Wingfield, Tun, \& McCoy, 2005) have suggested that even when the speech signal is correctly identified by listeners with a mild-to-moderate hearing loss, the perceptual and cognitive efforts required for successful word recognition may take a toll on processing resources that might otherwise be used for subsequent processing (e.g., for encoding the speech content into memory). Systematic research on the differential impact of perceptual and cognitive load in speech segmentation is only now emerging, and the consequences of such an approach seem to go far beyond the segmentation domain.

\section{AUTHOR NOTE}

Preparation of this article was supported by a postdoctoral grant from Fundação para a Ciência e a Tecnologia-Ministério da Ciência, Tecnologia e Ensino Superior to T.F. (SFRH/BPD/46979/2008), as well as by a joint grant from Fundação para a Ciência e a TecnologiaMinistério da Ciência, Tecnologia e Ensino Superior and European Community FEDER funding (Project PTDC/PSI/66077/2006, "Cognitive consequences of literacy"), and by Centro de Psicologia Clínica e Experimental-Desenvolvimento, Cognição e Personalidade of the Universidade de Lisboa, Portugal. The second author is Senior Research Associate of the Belgian Fonds de la Recherche Scientifique. The authors express their gratitude to the Psychological Department of the University of Evora and to Isabel Leite for all the help with the experimental procedures run there. We are also grateful to Sven Mattys and two anonymous reviewers for very important comments on a previous version of the manuscript. Correspondence concerning this article should be addressed to T. Fernandes, Speech Lab-Lab. de Fala, Faculdade de Psicologia e de Ciências da Educação da Universidade do Porto, R. do Dr. Manuel Pereira da Silva, 4200-392 Porto, Portugal (e-mail: tfernandes@fpce.up.pt).

\section{REFERENCES}

Astheimer, L. B., \& SANDERS, L. D. (2009). Listeners modulate temporally selective attention during natural speech processing. Biological Psychology, 80, 23-34. doi:10.1016/j.biopsycho.2008.01.015

BYRD, D. (1996). Influences on articulatory timing in consonant sequences. Journal of Phonetics, 24, 209-244.

ByRd, D., \& SAltzman, E. (1998). Intragestural dynamics of multiple prosodic boundaries. Journal of Phonetics, 26, 173-199.

Cho, T., \& McQueen, J. M. (2005). Prosodic influences on consonant production in Dutch: Effects of prosodic boundaries, phrasal accent and lexical stress. Journal of Phonetics, 33, 121-157. doi:10.1016/j.wocn .2005 .01 .001
Christiansen, M. H., \& Allen, J. (1997). Coping with variation in speech segmentation. In A. Sorace, C. Heycock, \& R. Shillcock (Eds.), Language acquisition: Knowledge representation and processing (pp. 327-332). Edinburgh: University of Edinburgh Press.

Cohen, A., Ivry, R. I., \& Keele, S. W. (1990). Attention and structure in sequence learning. Journal of Experimental Psychology: Learning, Memory, \& Cognition, 16, 17-30.

Colle, H. A., \& Welsh, A. (1976). Acoustic masking in primary memory. Journal of Verbal Learning \& Verbal Behavior, 15, 17-31.

CooKe, M. (2006). A glimpsing model of speech perception in noise. Journal of the Acoustical Society of America, 119, 1562-1573. doi:10.1121/1.2166600

CoOKe, M., Lecumberri, M. L. G., \& Barker, J. (2008). The foreign language cocktail party problem: Energetic and informational masking effects in non-native speech perception. Journal of the Acoustical Society of America, 123, 414-427. doi:10.1121/1.2804952

Davis, M. H., Marslen-Wilson, W. D., \& Gaskell, M. G. (2002). Leading up the lexical garden path: Segmentation and ambiguity in spoken word recognition. Journal of Experimental Psychology: Human Perception \& Performance, 28, 218-244. doi:10.1037/0096 $-1523.28 .1 .218$

Dutoit, T., Pagel, V., Pierret, N., Bataille, F., \& Van der VRECKEN, O. (1996). The MBROLA project: Towards a set of high quality speech synthesizers free of use for non commercial purposes. In ICSLP-1996, 1393-1396.

Fernandes, T., Ventura, P., \& Kolinsky, R. (2007). Statistical information and coarticulation as cues to word boundaries: A matter of signal quality. Perception \& Psychophysics, 69, 856-864.

FISER, J., \& AsLIN, R. N. (2001). Unsupervised statistical learning of higher-order spatial structures from visual scenes. Psychological Science, 12, 499-504.

FoDOR, J. A. (1983). The modularity of mind: An essay on faculty psychology. Cambridge, MA: MIT Press.

FoDOR, J. A. (2000). The mind doesn't work that way: The scope and limits of computational psychology. Cambridge, MA: MIT Press.

Fougeron, C., \& Keating, P. A. (1997). Articulatory strengthening at edges of prosodic domains. Journal of the Acoustical Society of America, 101, 3728-3740.

Gow, D. W., \& GoRdon, P. C. (1995). Lexical and prelexical influences on word segmentation: Evidence from priming. Journal of Experimental Psychology: Human Perception \& Performance, 21, 344-359.

Hauser, M. D., Newport, E. L., \& Aslin, R. N. (2001). Segmentation of speech stream in a non-human primate: Statistical learning in cotton-top tamarins. Cognition, 78, B53-B64.

Jacquemot, C., Dupoux, E., Decouche, O., \& Bachoud-Lévi, A.-C. (2006). Misperception in sentences but not in words: Speech perception and the phonological buffer. Cognitive Neuropsychology, 23, 949-971. doi:10.1080/02643290600625749

JOHNSON, E. K., \& JUSCZYK, P. W. (2001). Word segmentation by 8-month-olds: When speech cues count more than statistics. Journal of Memory \& Language, 44, 548-567. doi:10.1006/jmla.2000.2755

JONES, D. [M.] (1999). The cognitive psychology of auditory distraction: The 1997 BPS Broadbent lecture. British Journal of Psychology, 90, 167-187.

Jones, D. M., Macken, W. J., \& Murray, A. C. (1993). Disruption of visual short-term memory by changing-state auditory stimuli: The role of segmentation. Memory \& Cognition, 21, 318-328.

Kirkham, N. Z., Slemmer, J. A., \& Johnson, S. P. (2002). Visual statistical learning in infancy: Evidence for a domain general learning mechanism. Cognition, 83, B35-B42.

KLATT, D. H. (1980). Speech perception: A model of acoustic-phonetic analysis and lexical access. In R. A. Cole (Ed.), Perception and production of fluent speech (pp. 243-288). Hillsdale, NJ: Erlbaum.

KüHNERT, B., \& NOLAN, F. (1999). The origin of coarticulation. In W. J. Hardcastle \& N. Hewlett (Eds.), Coarticulation: Theory, data, and techniques (pp. 7-30). Cambridge: Cambridge University Press.

Liberman, A. M., \& Studdert-Kennedy, M. (1978). Phonetic perception. In R. Held, H. Leibowitz, \& H.-L. Teuber (Eds.), Handbook of sensory physiology: Vol. 8. Perception (pp. 143-178). Berlin: Springer.

Marsh, J. E., Hughes, R. W., \& Jones, D. M. (2009). Interference by process, not content, determines semantic auditory distraction. Cognition, 110, 23-38. doi:10.1016/j.cognition.2008.08.003

MatTys, S. L. (2004). Stress versus coarticulation: Toward an inte- 
grated approach to explicit speech segmentation. Journal of Experimental Psychology: Human Perception \& Performance, 30, 397-408. doi:10.1037/0096-1523.30.2.397

Mattys, S. L., Brooks, J., \& Cooke, M. (2009). Recognizing speech under a processing load: Dissociating energetic from informational factors. Cognitive Psychology, 59, 203-243.

Mattys, S. L., White, L., \& Melhorn, J. F. (2005). Integration of multiple speech segmentation cues: A hierarchical framework. Journal of Experimental Psychology: General, 134, 477-500. doi:10.1037/0096 -3445.134.4.477

McCoy, S. L., Tun, P. A., Cox, L. C., Colangelo, M., Stewart, R. A., \& WingFIELD, A. (2005). Hearing loss and perceptual effort: Downstream effects on older adults' memory for speech. Quarterly Journal of Experimental Psychology, 58A, 22-33. doi:10.1080/02724980443000151

McQueen, J. M., Norris, D., \& Cutler, A. (1994). Competition in spoken word recognition: Spotting words in other words. Journal of Experimental Psychology: Learning, Memory, \& Cognition, 20, 621-638.

Mirman, D., McClelland, J. L., Holt, L. L., \& Magnuson, J. S. (2008). Effects of attention on the strength of lexical influences on speech perception: Behavioral experiments and computational mechanisms. Cognitive Science, 32, 398-417. doi:10.1080/03640210701864063

Neath, I., Guérard, K., Jalbert, A., Bireta, T. J., \& SurpreNANT, A. M. (2009). Irrelevant speech effects and statistical learning. Quarterly Journal of Experimental Psychology, 62, 1551-1559. doi:10.1080/17470210902795640

Norris, D., McQueen, J. M., \& Cutler, A. (1995). Competition and segmentation in spoken-word recognition. Journal of Experimental Psychology: Learning, Memory, \& Cognition, 21, 1209-1228.

Pacton, S., \& Perruchet, P. (2008). An attention-based associative account of adjacent and nonadjacent dependency learning. Journal of Experimental Psychology: Learning, Memory, \& Cognition, 34, 80-96. doi:10.1037/0278-7393.34.1.80

Perruchet, P., \& PACton, S. (2006). Implicit learning and statistical learning: One phenomenon, two approaches. Trends in Cognitive Sciences, 10, 233-238. doi:10.1016/j.tics.2006.03.006

Rowland, L. A., \& Shanks, D. R. (2006). Attention modulates the learning of multiple contingencies. Psychonomic Bulletin \& Review, 13, 643-648.

Saffran, J. R., Aslin, R. N., \& Newport, E. L. (1996). Statistical learning by 8-month-old infants. Science, 274, 1926-1928.

Saffran, J. R., NewPort, E. L., \& Aslin, R. N. (1996). Word segmentation: The role of distributional cues. Journal of Memory \& Language, 35, 606-621.

Saffran, J. R., Newport, E. L., Aslin, R. N., Tunick, R. A., \& BarRUECO, S. (1997). Incidental language learning: Listening (and learning) out of the corner of your ear. Psychological Science, 8, 101-105.

Salverda, A. P., Dahan, D., Tanenhaus, M. K., Crosswhite, K., Masharov, M., \& McDonough, J. (2007). Effects of prosodically modulated sub-phonetic variation on lexical competition. Cognition, 105, 466-476. doi:10.1016/j.cognition.2006.10.008

Schneider, W., Eschman, A., \& Zuccolotto, A. (2002a). E-Prime reference guide. Pittsburgh: Psychology Software Tools, Inc.
Schneider, W., Eschman, A., \& Zuccolotto, A. (2002b). E-Prime user's guide. Pittsburgh: Psychology Software Tools, Inc.

Shanks, D. R., Rowland, L. A., \& Ranger, M. S. (2005). Attentional load and implicit sequence learning. Psychological Research, 69, 369382. doi:10.1007/s00426-004-0211-8

SNODGRAss, J., \& VANDERWART, M. (1980). A standardized set of 260 pictures: Norms for name agreement, image agreement, familiarity, and visual complexity. Journal of Experimental Psychology: Human Learning \& Memory, 6, 174-215.

SwINGLEY, D. (2005). Statistical clustering and the contents of the infant vocabulary. Cognitive Psychology, 50, 86-132. doi:10.1016/j.cogpsych 2004.06.001

Thiessen, E. D., \& Saffran, J. R. (2003). When cues collide: Use of stress and statistical cues to word boundaries by 7- to 9-month-old infants. Developmental Psychology, 39, 706-716. doi:10.1037/0012 $-1649.39 .4 .706$

Toro, J. M., SinnetT, S., \& Soto-Faraco, S. (2005). Speech segmentation by statistical learning depends on attention. Cognition, 97, B25-B34.

Turk-Browne, N. B., Jungé, J. A., \& Scholl, B. J. (2005). The automaticity of visual statistical learning. Journal of Experimental Psychology: General, 134, 552-564. doi:10.1037/0096-3445.134.4.552

Wingfield, A., Tun, P. A., \& McCoy, S. L. (2005). Hearing loss in older adulthood: What it is and how it interacts with cognitive performance. Current Directions in Psychological Science, 14, 144-148.

YANG, C. D. (2004). Universal Grammar, statistics or both? Trends in Cognitive Sciences, 8, 451-456. doi:10.1016/j.tics.2004.08.006

\section{NOTES}

1. Coarticulation is usually defined as a change in the acousticphonetic content of a speech segment due to anticipation or preservation of adjacent segments (e.g., Kühnert \& Nolan, 1999). The extent of coarticulation between adjacent segments is influenced by the presence of a prosodic boundary (e.g., Byrd, 1996; Byrd \& Saltzman, 1998). This general segment strength (Cho \& McQueen, 2005) can convey information about the local coherence versus disjuncture in connected speech (Fougeron \& Keating, 1997).

2. TPs are operationally defined as the probability with which one syllable $(x)$ predicts the following one $(y): \operatorname{TP}(y \mid x)=$ frequency $(x y) /$ frequency $(x)$. In any natural language, TPs tend to be higher when the two syllables follow one another within a word than when they straddle a word boundary (Swingley, 2005; but see Yang, 2004).

3. According to Mattys et al. (2009), another reason might be that the statistically defined "words" used by Fernandes et al. (2007) occurred many times in the speech stream, as it is usual in ALL settings. This may have contributed to improve their intelligibility over the course of the familiarization phase, ultimately outweighing difficult-to-perceive coarticulatory cues.

(Manuscript received April 26, 2009; revision accepted for publication April 3, 2010.) 